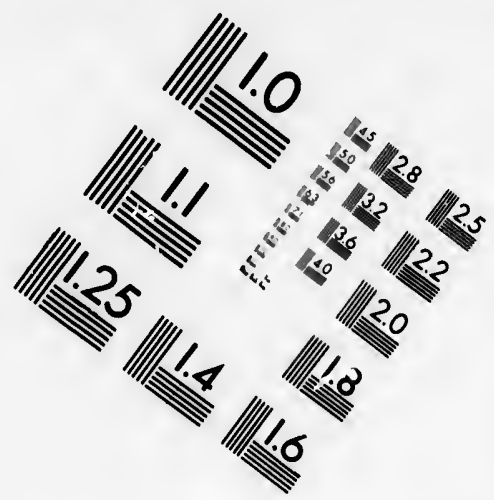

TEST TARGET (MT-3)
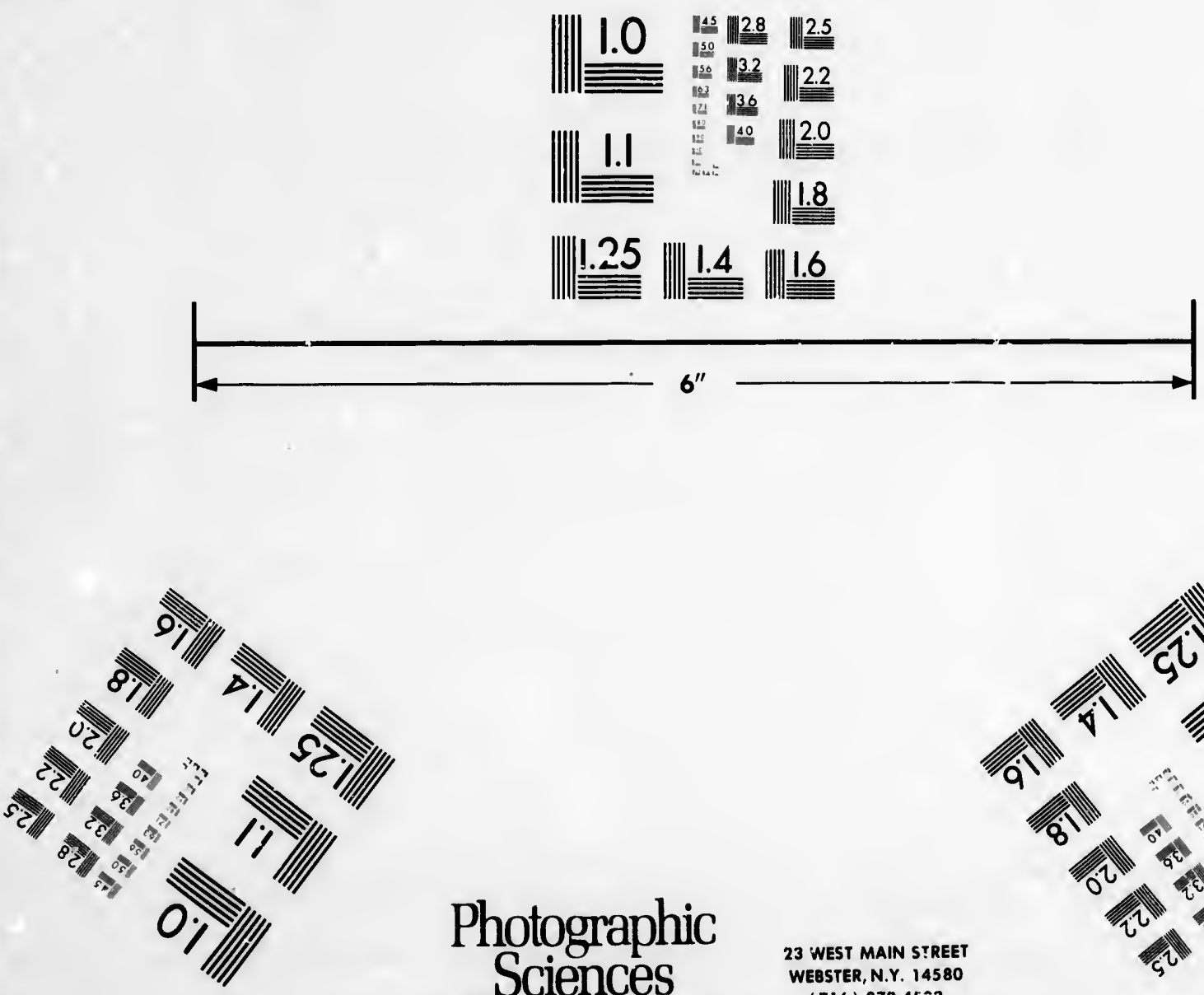

Photographic Sciences Corporation
23 WEST MAIN S!REET WEBSTER, N.Y. 14580

(715) 872-4ㄴ:3

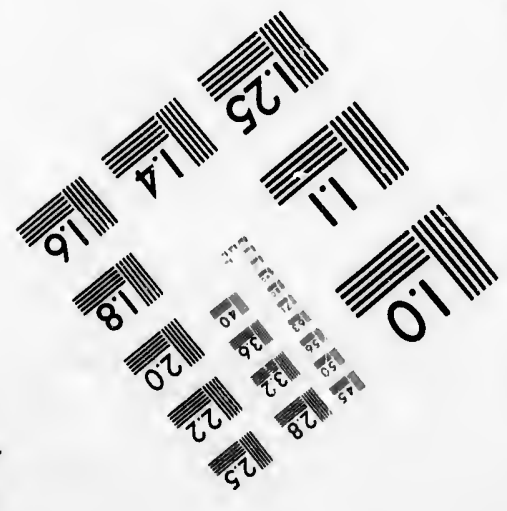




\section{CIHM/ICMH Microfiche Series.}

\section{CIHM/ICMH Collection de microfiches.}


The Institute has attempted to obtain the best original copy available for filming. Features of this copy which may be bibliographically unique. which may alter any of the images in the reproduction, or which may significantly chenge the usual method of filming, are checked below.

Coloured covers/

Couverture de couleur

\section{Covers damaged/}

Couverture endommagée

Covers restored and/or laminated/

Couverture restaurée et/ou pelliculée

Cover title missing/

Le titre de couverture manque

Coloured maps/

Cartes géographiques en couleur

Coloured ink (i.e. othar than blue or black)/

Encre de couleur (i.e. autre que bleue ou noire)

Coloured plates and/or illustrations/

Plar.ihes et/ou illustrations en couleur

Bound with other material/

Relié avec d'autres documents

Tight binding may cause shadows or distortion along interior margin/

La reliure serrée peut causer de l'ombre ou de la distortion le long de la marge intérieure

Blank leaves added during restoration may appear within the text. Whenever possible, these have been omitted from filming/

II se peut que certaines pages blanches ajoutées lors d'une restauration apparaissent dans le texte, mais, lorsque cela était possible, ces pages n'ont pas été filmées.

Additional comments:/

Commentaires supplémentaires:
L'Institut a microfilıné le meilleur exemplaire qu'il lui a été possible de se procurer. Les détails de cet exemplaire qui sont peut-etre uniques du polnt de vue bibliographique, qui peuvent modifier une image reproduite, ou qui peuvent exiger une modification dans la méthode normale de filmage sont indiqués ci-dessous.

Coloured pages/

Pages de couleur

Pages damaged/

Pages endommagées

Pages restored and/or laminated/

Pages restaurées et/ou pelliculées

Pages discoloured, stail : 9 or foxed/

Pages décolorées, tachetíes ou piquées

Pages detached/

Pages détachées

Showthrough/

Transparence

Quality of print varies/

Qualité inégale de l'impression

Includes supplementary material/

Comprend du matériel supplémentaire

Only edition available/

Seule édition disponible

Pages wholly or partially obscured by errata slips, tissues, etc., have been refilmed to ensure the best possible image/ L6: pages totalement ou partiellement obscurcies par un ferillet d'errata, une pelure, etc., orit été filmées à nouveau de façon z. obtenir la meilleure image possible.

This item is filmed at the reduction ratio checked below/

Ce document est filmé au taux de réduction incíqué ci-dessous.

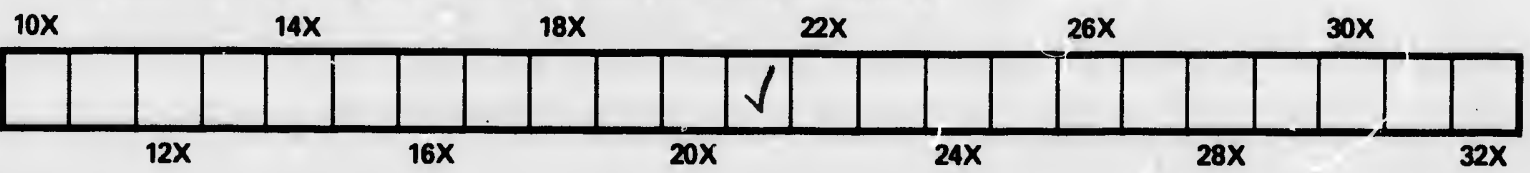


The copy filmed here has been reproduced thanks to the generoslty of:

\section{Library of the Public}

Archives of Canada

The images appearing here are the best quality possible considering the conclition and legibility of the orlginal copy and in keeplng with the filming contract specifications.

Original coples in printed paper covers are filmed beginning with the front cover and ending on the last page with a printed or illustrated impression, or the back cover when appropriate. All other original copies are filmed beginuing on the first page with a printed or Illustrated impression, and entire on the last page with a printed or illustrated : npros: on.

The last recorded frame on each microfiche shall contain the symbo: $\rightarrow$ Imeaning "CONTINUED"), or the symbol $\nabla$ (meaning "END"), whichover applies.

SAaps, plates, charts, etc., may be filmed at different reduction ratios. Those too large to be entirely included in one exposure are filmed beginning in the upper left hand corner, left to right and top to bottom, as many frames as required. The following diagrams illustrate the mothod:
L'exempleire filmé fut reproduit grâce à la gónérosité de:

La bibliothèque des Archives publiques du Canada

Les images suivantes ont été reproduites avec le plus grand soin, compte tenu de la condition et de la netteté de l'exemplaire filmé, et en conformité avec les conditicns du cuntrat de filmage.

Les exemplaires originaux dont la couverture en papier est imprimé sont filmés en commencant par le premier plat et en terminant soit par la dernière page qui compo.te une empreinte d'impression ou d'illustration, soit par le second plat, selon le cas. Tous les autres exemplaires origlnaux sont filmés en commençant par la première page qui comporte une empreinte d'impression ou d'illustration et en terminant par la derniére page qui comporte une telle empreinte.

Un des symboles suivants apparaîtra sur la derniére image de chaque microfiche, selon le cas: le symbole $\rightarrow$ signifie "A SUIVRE", Ie symbole $\nabla$ signifie "FIN".

Les cartes, planches, tableaux, etc.. peuvent être filmés á des taux de réduction différents. Lorsque le document est trop grand pour être reproduit en un seul cliché, il ust filmé a partir de l'angle supérieur gauche, de gauche à droite. et de haut en bas, en prenant le nombre d'images nécessaire. Les diagrammes suivants illustrent la mbthode.
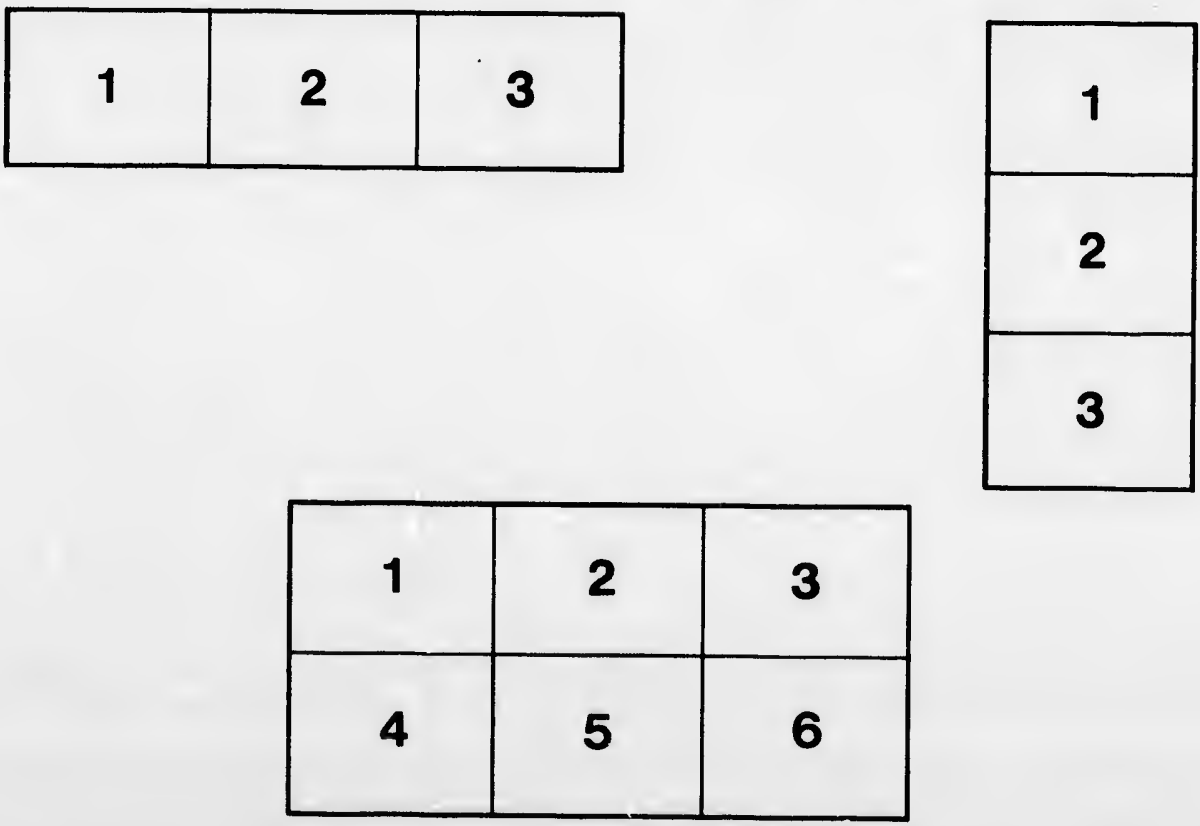
888

DEPARTEMENT DE

l'Agriculture et de la Colonisation

PROVINCE DE QUEBEC.

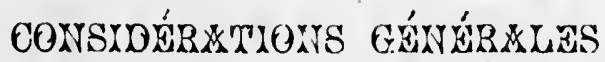

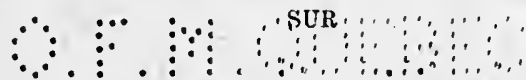 \\ LAGRICULTURE, LA COLONISATION, LE \\ RAPATRIEMENT ET L'IMMIGRATION, \\ PAR \\ LE REVEREND A. LABELLE. \\ Assistant-Commissaire.
}

(Extrait du kapport Officiel de Departkufint).

QUÉBEC:

1858. 
8

$+\cos x$

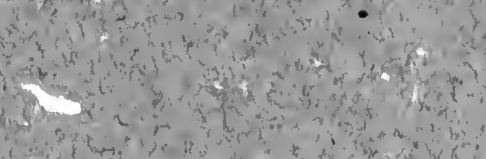

$$
\text { (4) }
$$

$$
\text { ine } x^{2} x^{2}
$$

$$
x^{2}=y^{2}
$$$$
\text { int }
$$$$
\text { in } y^{2} y
$$

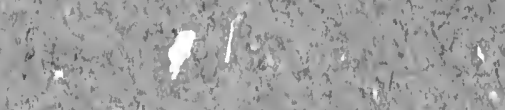

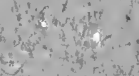

$$
\text { (3) }
$$$$
\text { ओ आ }
$$

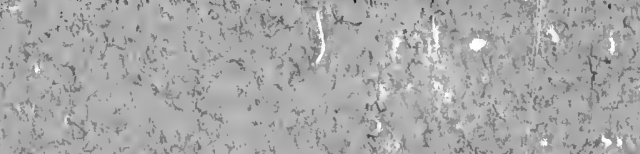

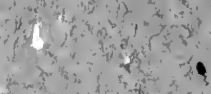

$$
\text { and }
$$$$
6 x^{2}
$$ 


\title{
DEPAYTEMENT DE
}

l'Agriculture et de la Colonisation

\author{
PROVINCE DE QUÉRE.
}

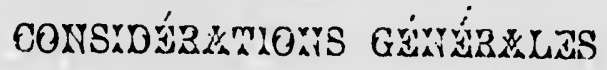 \\ $2 L^{2}$ \\ LAGRICULTUKE. LA COLUASATION, LE \\ RAPATRIEMENT ET LIMMITRATION, \\ PAR \\ Le reverend a. LABElle. \\ Assistant-Commissaive. \\ (Extrait du fiaport Orficipl de Départeyext).
}

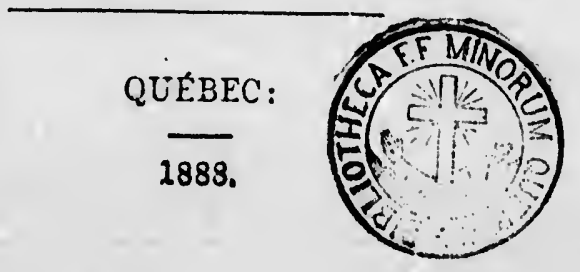




\section{8 \\ (82)}

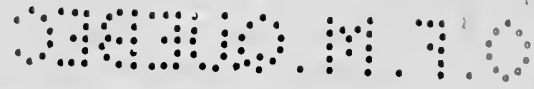




\section{I. $\triangle$ GRICULTURE}

In province de Quévec est ẹsentiellement agricole. Iat plus grande partie de sil population se livre it cette noble profession qui fait les races fortes et viriles. Tuut l'y invite: les traditions de ses pières, les belles plaines du Suint-Laurent, de l'Ottawa, du Richeliew. les vastes territoires des eantons du Nord et du Suld si magnifiquenent arrosés et si fertiles.

Les innombrables ponvoirs: hydraliques de cette province, sès rrichesses' minièr'es et forestières, sit proximité des ports maritimes pour y diriger facilement ses produits par les chemins de fer et la grande voie fluviale du Saint-Lanrent, it nulle autre pareille dans le monde, lui donnent des avantages inappréciables. Elle est done admirablement située pour que l'agriculíre et l'industrie s'unissent afin d'alimenter ses marehés locaux et śtrangers et de résondre le grand problème de la force unie à la richesse. Elle occupe aussi une position stratégique dans la confédération et devient, de ce fait, un pivot essentiel dans la grande machine gouvernementale. On ne pent cionc y toucher imprudenment ou l'affaiblir sar.s mettre en danger toute l'économie sociale de la Puissance. 
Toute nation qui fait de 'l'agriculture sa principale occupation conserve toujours un degré de vitalité et do santé qui lui assure l'avenir.

L'ouvrier peut gagner parfois plus d'argent que le cultivateur, mais la constitution du premier se détériore au travail délétère des fabriques et l'affaiblissemment de ses descendants qui suivent la même corrièréc ne fait qụ̂ progresser de générations en générntious et entrafner les maux lamentables qui sont la plaie des pays manufacturiers.

Il est donc du devoir du gouvernement, puisque ses domaires agricoles sont immenses, de favoriser l'agriculture par tous les moyens a sa disposition et de pousser

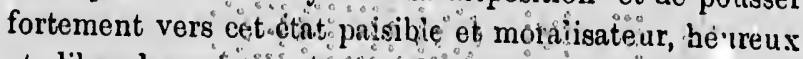
et libre, les sujetśqui sónt placés sous sa juriảiction, en se rappelant que la conquête du monde fut l'apanage cles soldats agriculteurs, les Romains.

Pour rendre cette profession attrayante, it faut la rendre payante et faire connaitre tous les secrets de l'art agricole sous toutes ses formes.

Divers moyens sont suggérés qui sont excellents, qui tendent tous au même but et que l'on doit favoriser graduellement, svivant les temps, ies lieux, les circonstances, les habitudes des gens et les ressources du gouvernement.

Il est certain que l'agriculture a fait de grands progrès dans la province de Québec sous l'impulsion du Conseil de l'agriculture qui sans cesse doit perfectionner et multiplier ses moyens d'action. 
Nous possédons beaucoup d'agronome, des plus distingués, comparables aux meilleurs agronomes do tout autre pays, mais, il faut l'avouer, un grand nombre d'agriculteurs n'ont pas toujours profité des efforts cilı gouvernement pour les diriger dans la bonne voie, les uns par une coupable indifférence, les autres, pur un attachement regrettable $\dot{a}$ de vieilles routines. C'est cette classe qu'il faut atteindre, avec l'aide du temps, en déployant du couruge et de la persévérnnce, qui vaine tous les obstacles. Que l'on sache qu'il faut ringt aus pour élever" lin enfant et cent ans pour refaire une nation.

La société change tons les jours de face par ses facilités de communication, ses uouveaux marchés de l'intérieur et de l'extérietir, et, par lá même, uno culture qui aujourd'hui est plus lucrative peut demain le devenir moins.

Notre pays a done subi l'influetice de ces modifications commerciales qui affectent les conditions écovomi ue's de toute nation et dont il falit savoir profiter.

La fécondité des nouvelles terres qui rendent longtemps avec usure la semence qui leur est confiée, et presque sans effort d'intelligence de la part du cultivateur, parce que le sol est enrichi avec excès depuis le délıge par l'humus des plantes et des arbres, a fiit croire malheureusement que la terre ne pouvait s'épuiser par des récoltes successives de grain sur grain. 
L'agriculteur ne reféchissait pas que lo sol, en nous donrant ses moissons luxuriantes, perdait peu à peu seз éléments fertilisants, et que lo terrain ne pouvait renou. veler ses forces vives do fócondité sans l'eugrais qui cst lo pain nourrieier de la terre.

L'Iown, qui a donné jusqu'à 25 à 30 minots de bló par acre, no rend plus, terme moyen, que 8 ì 9 minots. C'est done une loi de la nature que l'ọn ne peut mépriser saus de tristes mécomptes."

$\Lambda$ l'heure qu'il est, cette erreur a' disparu de l'esprit de notre population et beaucoup do cultivateurs comprennent que le fumier est uno mine d'or pour eux.

Cet axiome de la valeur des fumiers étant bien eoinpris partout, lo reste pour ainsi dire viendra par sureroit. En Belgique, le pays lo mieux cultivédu monde, la richesse du cultivateur s'estime par l'amas d'engrais que l'on remarque devant ses constrnctions agricoles.

Heureusement que le cultivateur a commeneć par adopter un bon système de rotation, ì semer des graines propres à améliorer ses pâturagres et ses fourrages, à faire plus de culture nettoyante pour sareler sa terre, à augmenter le nombre de ses animaux pour accroitre la quantité de ses fumiers. Plusieurs, en outre, emploient les amendements et engrais minéraux, comme la chaux, les cendres, et surtout le plâtre et le phosphate de chanx. C'est encore un progrés ì constater.

Ces connaissances pénètrent de plus en plus dans le 
peuple et j'espicre les répandre avec plus d'efficncitú si lo gouvernement le désire, par l'encouragement des cer'sles agricoles, et le meilleur fonctionnement des Suciétís d'agriculture, par des conférences sur la bonne culture, par l'amélioration de nos écoles, par une plus grando diffusion du Journal de l'Agriculture, par la création d'une station expérimentale et laboratoire de chimie agricole, ot, parmi les cultivateurs, d'une classo d'homneur qui sera comme le sénat de l'ngriculture.

Te compte aussi sur le bon exemplo des fermes d'Oka it i'Arundel. Ce sont les mémes hommes qui ont créú en Frinse, ì Belle-Fontaine, ì St-Laurent sur Sévres, cue cúlébres métairies que tout le monde admire. l'umrịuoi ces religieux u'auraient-ils pas les mêmes succèj ici qu'en France? Ne serait-il pas à propos de les ripundre avec le temps ot les circonstances dans les différentes parties de la province? On peut en dire autant de la ferme des sourds-mucts, près de Montréal, que le Conseil d'agriculture a recommandée d'une manière particulière à lattention du gouvernement. .

A mon a vis, l'amélioration agricole la plus importante, par ses conséquences immédiates, qui ait'été accomplie dans la province de Québec est l'établissement des beurreries et des fromageries, au nombre d'environ 672. On compte plus de beurreries, dit-on, dans notre proviuce que dans Ontaric. C'est un bon point en notre faveur. Quand on pense qu'nne vache ordinaire, avec des soins ordinaires, donne un revenu annuel de $\$ 25$, en beurre, ou fromage et viande, un mouton, $\$ 3$ à $\$ 4$. 
quel est le cultivateur qui ne pourrait pas, relativement it peu de frais, se tailler un domaine dans les Laurentides ou les cantons du sud, et se créer un troupeau de quinze à vingt vaches, bonnes laitières, et de 40 \ 50 moutons, sans parler de le récolte de ses grains, légumes et fourrages? Il n'y a iqu'it $y$ pcnser pour le vouloir. Nous exportons your $\$ s, 000,000$ de beurre et de fromage en Europe. C'est une exportation qui peut être doublée, triplée en peu d'années. Nos fromages sont cotés au plus haut prix sur les marcliés anglais et belges. Notre beurre n'a pas la même réputation et il ne tient qu'à nous de l'obtenir.

Si nous le désirons fermement, notre beurre pourrn rivaliser avec celui du Danemark, de la Hollande ou de la Normandie, comme nous le faisons pour le fromage. Dans ces matières et dans les mêmes conditions, je ne císespère pas d'atteindre à la perfection des autres nations: pour cela il nous faudrait un inspecteur de beurre parfaitement qualifié et d'une réputation inattaquable, qui, par un examen sévère et intelligent, cuonnerait une bome réputation à ce produit si inportant de nos fermes.

Il est prouvé par des faits indéniables que l'éle vage des bestianx, (à moins diz cultiver près des villes) pour la falorication du beurre es. du fromage, est l'exploitation ngrieole la plus payante. J'ai toujour's remarqué que le cultivnteur qui s'y livrait avee patience et intelligence s'eurichissait ì vue d'ceil, tandis que ses voisins, qui 
sulvaient les vieilles routines, végétaient autour de lu?. Lo premier multipliait avec sucrès ses animaux, et par là même, l'ahoudance de ses eugrais, lesquels décupjaient la capacité productive de son sol pour les grains, les légumes et les fourrages. L'objection des longs hiveris est résolue par la construction des silos, et je no donte pas que celui qui adopte ce mode d'utiliser ses fourrages, en recueillera les plus grands profits, comme nous l'attestent les meilleurs agronones.

Il ne serait pas hors de propos de farler de l'améliora. tion de la race cievaline. Le Canadien aime le cheval, et souvent neglige les autres animanx pour nourrir le cheral avec soin. quoique eette industrie agricole mo paraisse moins lucrative que celle des vaches laitières. Cependant, il serait bon de protiter des goûts et des aptitudies de la population paur l'élevage des chevaux.

Le gouvernement pourrait aider ceux qui seraient disposés à étriblir des haras, ou retenir une certaino soimne stir les tonds agrícoles, acheter lui-même des sheraux et los répandre daus la province, comme le fair annullement, uns province maritime, lo Nouveau. Brunswicl:

Quant a Cexposition provinciale quì devait se tenir ¿Montréal durant le cours de l'été, si elle n'a pas eu lieu, c'est que la municipalité cie cette ville n'a pas offert au gonvernement dè contzibuer dians les dépenses pour une somme égate a celle que celui-ci avait mise dans les estimations budgétaires. 


\section{II. COLONISATION.}

La colonisation et l'agriculture sont pour ainsi dire la suême chose. Elles joutent le rôle principai dans l'augmentation de la richesse et de la prospérité de cetto province. Cependant je puis dire que la colonisation de nos terres incultes doit occuper la première place dans notre esprit.

Notre influence dans les conseils des Chambres législatives dépend de notre accroissement par la colonisation ; c'est en effet celle-ci qui résoudra les graves questions du présent et de l'avenir. Chaque pas fait dans la forêt par le défrichement' est une conquête qui fortifie nos Uroits, nos institutions et nos lois. "Emparons-nous dn sol" est un motto qui devrait être écrit en lettres d'or au-dessus de la porte de chaque maison. Négliger l'étendre la colonisation, c'est porter un coup fatal an pays et détruire l'œuvre glorieuse de nos ancêtres.

En retardant d'ouvrir, par des mesures énergiques, soit volontairement, soit involontairement, nos terres incultes à la colonisation, nous avons contribué plus qu'on ne pense à pousser notre population à émigreı aux Etats-Unis. Quo voulez-vous faire ? Un agricul. 
teur, à la tête d'une famille de 5 ou 6 garçous, aurait bien voulu les établir dans leur paroisse natale, nnais les propriétés étaient d'un prix trop élevé. A peine ses revenus suffisaient-ils à naurrir et à élever sa nombreuse progéniture. Les fabriques étaient remplics d'ouvriers et les magasins de commis. Ce bon père regardait d'un œil attristé l'immense région de nos bonnes terres incultes, mais cette région lui-était fermée par le inauque de routes et de chemins de fer. Se faire homme de peine, se soumettre à ce triste régime, c'était trop pénible pour lui, surtout dans son propre pays. Il ne restait plus d'autre alternative d̀ ses enfants que de prendre le chemin des Etats sillonnés de chemins de fer, où l'industrie marchait à pas de géant et réolamait l'cmploi inmédiat de bras innombrables. Il faut ajouter que jour un certain nombre une mauvaise culture, le luxe, les folles dépenses, quelques fois, bélas ! l'ivrognerie, un tempérament aventureux que nous tenons des Normands, ont pu accélérer cet exode de nos nationaux. Il n'y a d'autre moyen de réagir contre ce courant qui now décime que de pousser fortement notre population à coloniser surtout dans ce temps oủ le Canada offre, pour y vivre, autant d'avantages que les Etats-Unis.

Façonuée à la vie dure et pénible des champs, la race canadienre possècie des aptitudes particulières pour coloniser.

Il est vrai que la colonisation a fait de grands progrès, depuis un certain nombre d'années, grâce aux cheming 
12

que le gouvernement a construits dans la forêt vierge, ez ('ll cépit des difficultés légales suscitées sur les pas du colun. Mais ce n'est pas encore assez. Que de colons sunfrent depuis longtemps de l'absence de moyers do communication:

Le véhicule par excellence de la colonisation, ce soint les chemins de fer qui changent la face d'un pays comme Inil elichantement. Auss le colon, qui est le principal fucteur de l'avancement du pays, les demande à grancis cris. Et comment pourrait-il, dans les commencements, transporter le lard, la fleur, dont il a besoin à son domicile, quand il vit ì 30 ou 40 lieues des chemins de f: 1 ei qu'il n'a d'autre moyen do transport que sa charrette ou son traîneau.

C'est done le moment favorable de mettre cette politique grénéreusement en pratique, dût la province s'endetter de plusieurs millions, et quand bien même il faudrait hausser le prix des terres dont on décuplerait la valeur par les voies ferrées. D'ailleurs, en 1882, lo colon n'a-t-il pas envoyé à la Chambre des requêtes cleinandant de faire payer les lots $\$ 1.00$ l'acre, pourvil qu'ou lui fît des chemins de fer ? Combien n'y a-t-il pas de colons qui préfèreraient payer leurs terres une piastre l'acre, avec des chemins de fer à proximité, que de les recevoir gratuitement sans chemins de fer ?

Monsieur Ramean, ce véritable ami du Canada, conseille de faire des chemins.de fer danș l'intérieur, même en nous eudettant. 
Nous pouvons distinguer clans le Nord quatre grandes zônes qui offrent de rastes teritoires it colouiser:

La vallée du Téniscamingue, ì liqquelle des vaisseaux et un chemin de fer,.gui doit se prolonger jusqu'it Mattawa, donnent une valeur qu'on ne peut trop apprécier, et oú l'on pent établir 40 i 50 paroisses dans les meilleurs terrains de la jrovinee, en particulier an ratpicle des Quinze, près d'une riche mine de galène ar'gentifère que l'on exploite avec profit ;

$\dot{\mathrm{L}}_{\mathrm{a}}$ vallée proprement dite de l'Ottawa, où les chemins de fer de la Gatineau et du Montréal et Occidental doivent se raccorder en haut de cette rivière et se continuer jusqu'au Témiscamingue, toujous en traversant des terres des plus propres ì la colonisation, entre autres les bassins supérieurs de la Rouge, de lir Lièvre, de la Gatineau et de l'Ottawa : c'est un vaste champ de colonisation pour les districts de Montréal et d'Ottawa; car il est bon de se rappeler que le dernier colon est déjà fixé à 50 lieues de Hull et de Saint-Iérôme. C"est pour cette raison que le gouvernement a ouvert un grand chemin de colonisation partant du canton de Hartwell et rejoignant le chemin Chapleau sur le. lac Nominingue, afin de favoriser autant que possible l'établissement de cette immense région. Il est it propos de mentionner à ce sujet que Monsieur Arthur Buies a fait, dans le cours de l'été dermier, une excursion dans la vallée infúrieure de l'Outaouais, accompagnó d'un plhotographe chargu' de prendro des vues, afin d'y 
puiser les matériaux uécessaires à la publioation d'uno monographie de cette fertile partie de la province. Cette monographie devra paraftre à la stite d'un volume sur le bassin supérieur de l'Outaouais, volume qui est actuellement sous presse et qui est une description compliete de la nature, de la physionomie et des ressonices de ce prays is plein d'avenir:

La vallée du Saint-liaurice, à laquelle le chemin de fer des Trois-hivières doit donner une grande impulsicy colonisatrice et doit permettre de se relier en peu d'années aux ètablissements de la Rouge et du lac Témiscamingue. De ce grand lac all Saint-Maurice, nous pouvons coloniser jusqu'ä une distance de 60 lieues du Saint-Laurent et de l'Ottawa, parfois même bien audela. Nous pouvons aussi placer, par cantons successifs, des millions d'habitants, souvent sur des terres comparables a celles qui bordent le Saint-Laurent. Hélas! a peine le cinquièrne de ce territoire est-i! occupé !

Que dirons-nous en outre in chemin de fer du lac Saint-Jean jusqu'd Chicoutimi et de ceiui des Basses Laurentides, qui tous deux vont donner une impulsion mcalculable à la population de la vallée de ce magnifique. lac dont les bonnes terres s'étendent au loin dans l'intérieur? C'est lá que les habitants du district de Québec: trouveront de beaux héritages pour leurs enfants ; c'est lì que les appellont dés aujourd'hui des parents et des amis qui ont créé nombre d'établissements déià prospères. 
15

J'attire l'attention du gouvernement sur la purte est du lac où les terres de la plaine sout remarquables par la qualité, le nombre et l'étendue. Il faut tenter do nouveaux efforts pour peupler de braves gens cette immense région qui est l'arrière pays de Québec. Cette ville de Québec a fait noblement son devoir en dotant la Compagnie du lac Saint-Jean. D'autres villes pourraient suivre son exemple et peupler de la même façon leur pays d'intírieur; elles n'en auraient que plus d'honneur et trouveraient tout a $\mathrm{y}$ gagner pour leur crédit et leur commerce.

Iiappelons enfin la Gaspésie, les cantons de l'Est, lo comté de Bonaventure, et la fertile vallée de la Chandière. Lì aussi, nous devons apporter ì la colonisation toute l'aide possible en complétant les chenins de fer qui y sont ébauchés, car il y reste encore une ample quantité de bonnes terres à livrer aux lléfricheurs.

Il ne faut pas oublier que la Gaspésie notamnent contient un très grand nombre d'excellentes terres quii, partant des rivages du golfe Saint-Laurent ou de la baic des Chaleurs, se prolongent jusqu'à une distance de 20 à 30 milles dans l'intérieur. Voilà pourquoi le gouver. nement a fait entreprendre une exploration s'étendant depuis New-Richmond jusqu'au bassin de: Gaspé, afin de déterminer l'ouverture d'un chemin de colonisation av. sein des belles terres de cette contrée.

Quant à la vallée de la Mítipédiac, qui sécend entro les comtés de Rimousli et de Bonaventure, dans le vol- 
sinage immúdiat do ln Grapérie, sa fertilité ess trup connue pour qu'il nous soit !écessatire d'y attirer spécialement l'attentiou.

Il faut diriger nos colons vers ces terres nouvelles qui offrent infiniment plus de ressuurces que l'existence aléatoire de la pêche.

Le gonvernement vait donc d'un bon reil toutes ces entreprises de chemin de fer, puisqu'il octroie des terres et de l'argent pour les faire réussir, et je dois ajouter que lorsque ces voies ferrées seront finies, des millions et des millions s'ajouteront it la richesse mationale, et c'est alors que la colonisation, prenant an essol inoun, nous grandira dans le présent et assurem notre avenir, pourvu qu'on ne veuille pas faire tont a ln fois. que l'on procede avec mesure et que l'or saclie alle: graduellement pour atteiudre à l'ensemible. 


\section{III.}

\section{LE RAPATRIEMEN'T}

Voild une question qui s'agite depuis longtenıs, ot qui est de nature à flatter les sentiments les plus nobles et les plus légitimes de nos compatriotes.

Malgré les plus vifs désirs de voir se réaliser ce projet que caressait avec plaisir mon imagination, j'en suis venu à la conclusion, après de nombroux efforts tenté.s coucurreminent avec le rév. P'ère Fournier, résidant aux Etats-Unis, que ce mouvement ne pouvait: pas se faire avec le succès que j'arais rèvé.

Les Canadieñs fixćs aux Etats-Unis peuvent être classés en trois catégories: ceux qui sont riches ou vivent avec aisance; ceux qui sont nés aux Etats et sont accoutumés à la vie des manufactures; ceux qui sont des cultvateurs originaires du Canada, ont vécu au pays, et pleurent la patrie absente.

Quant aux deux premières classes, nous les recevro:s toujours ì bras ouverts, mais il ne serait pas prudent de les déranger de leurs occupations lucratives ou d'un état auprès duquel celui de défricheur parait beaucoup trop" dur. " Ce sont des frères qui perpétnent nos traditions avec honneur allx États-Unis: ils ont toujours une grande place dans notre cceur! 
Orant à la irrigienc clisse qui mérite anssi toute notre affection, nous n'avons pas oublié ses aspinations patriotiques vers nous. C'est pour cela que nous avons envoyé des agents aux États-Unis pour attirer l'attention de ces braves gens sur leur pays d'origine, et les inviter i y rentrer en leur signalant les avantages que va nous donner l'extension des chemins de fer dans nos terres colonisables. 
19

IV.

IMMIGRA'TION

Notre mission, en qualité de Canadiens, est d'établir la moitié de l'Amérique Septentrionale, puisune c'est lì l'útendue qu'occupe le Dominion. Il y aura là, uL jour, place pour une nation de 50 millions d'hommes.

Ia province de Québec, à l'heure qu'il est, à pcu de chose pres, est aussi grande que la France, et c'est ì peine si un quinzième de son territoire est peuplé.

- A l'exemplo de la Russie, nous deviendrons la nation du Nord, composée de différents éléments, greffée sur le tronc canadien et nourrie de la même sève. Le nom canadien flatte mon oreille, réjouit mon âme quand je pense au passé, au présent et à l'avenir. Toutes les nations européennes ont été formées de différents peuples qui, dans le cours dẹs siècles, sont devenus homogènes sous le même nom, tout en gardant les traces typiques de leur origine : exemple, les peuples de l'Angleterre ct de la. France dans lles veines desquels coule le sang de plusieurs races.

Il est impossible, par notre propre force vitale, d'établir rapidement cet immense pays, de profiter de suite de ses ressources extraordinaires, sans appeler à notre 
secours, comme lont fait les États-Unis, le surplus des populations de l'Eurole, chez lesquelles bon nombre d'habitants, mêmes riches, sont fatigués des crises périodiques qui chassent le konheur du foyer et sont tourmentés par les inquiétudes que font maitre en eux les révolutions, les guerres et les grèves qui sont toujours ì l'état latent. I. Canada leur offre un des meilleurs champs du monde pour jouir de la vie heureuse, calme et paisible, pour placer avec sécuritó leurs capitaux et exercer leurs talents, leurs aptitudes pour le commerce, les arts et métiers, er particulièrement pour l'agriculture.

Voila pourquoi nous faisons notre part dans ce travail d'immigration, et je dois dire que, relativement à l'argent que nous dépensons dans ce but, le succès couronne nos efforts, grâce au travail intelligent de nos agents d'immigration : MM. Marquette, Bodard et Foursin.

Notre action serait plus efficace si la Chambre, considérant les graves questions d'avenir qui sont en jeu, voulait augmenter l'article du budget qui a trait ì cette partie de nos opérations.

Sur ce paint, nous n'avons pas, il est vrai, les avantages da gouvernement fédéral qui, par ses douanes, est payé en retour de ses dépenses d'au moins $\$ 4.00$ \$ $\$ 5.00$ par tête annuellement, tundis que nous, comme gouvernement, nous avons des dépenses à encourir sans profit direct.

L'augmentation du subside fédéral, à chaque-recensement, nous dédommageriit en partie de nos - sacrifices: 
mais comnie nous 12 devons pas y penser pour le mo ment, faisons toujour's notre devoir au sujet de l'immigration étrangère et espérons que, tôt ou tard, luvis recueillerons les fruits de notre bonne volonté.

Il faut dire que nous recherchons non pas la quantité mais la qualitó des immigrants, et surtout ceux «ıii peuvent disposer d'un petit capital et l'employer d l'ayri. culture.

J'ai l'honneur d'être, monsieur le ministre,

Votre très humble et obéissant serviteur,

A. LABELLE, Ptre., Assistant-Commissaite. 
